\title{
Servitização em uma empresa fabricante de aeronaves: a relação dos serviços com o desenvolvimento de produto
}

\section{Servitization in an airplane company: the relation between services and new product development}

Leandro Almeida ${ }^{1}$

Márcia Terra da Silva ${ }^{2}$

Paulo A. Cauchick-Miguel ${ }^{3}$
1 Universidade de São Paulo - USP leandro.almeida@embraer.com

2 Universidade de São Paulo - USP mtdsilva@usp.br

3 Universidade de São Paulo - USP cauchick@usp.br

\section{Resumo}

As decisões tomadas no processo de desenvolvimento de produtos (PDP) influenciam a oferta de serviços por empresas de manufatura, no entanto, a literatura ainda é escassa em compreender essa relação. Nesse sentido, o objetivo do presente trabalho é analisar a relação entre as fases do PDP e os serviços oferecidos em uma empresa do setor aeronáutico, por meio de um estudo de caso, com dados coletados por entrevistas semi-estruturadas e análise documental. Inicialmente o artigo apresenta as fases do PDP e, em seguida, as relaciona com os serviços analisados. Os resultados confirmam que estes serviços são influenciados pelas decisões das fases do PDP. As relações identificadas influenciam diversos aspectos dos serviços com relação ao próprio PDP, ao escopo dos serviços, e infraestrutura existente. A contribuição gerencial do presente trabalho é a identificação da necessidade de incluir formalmente no PDP os requisitos dos serviços ofertados desde as primeiras fases deste processo.

Palavras-Chave: Desenvolvimento de produto. Serviços relacionados ao produto. Empresas de manufatura. Serviços pós-venda. Servitização.

\begin{abstract}
The importance of the inclusion of services in manufacturing companies has grown over the last decades due to several changes in the business environment. Among the aspects related with the offer of services by manufacturing companies is the fact that services are influenced by the decisions taken during the product development process (NPD). However, the literature is scarce when it comes to comprehend how this relationship occurs. In this sense, the objective of this study is to analyze the relationship between the NPD process activities and services through a case study in a manufacturing company from the aircraft industry. This work summarizes the phases of NPD, the selected services, and the relationship between NPD and these services. The results confirmed that the services are influenced by the decisions in taken the NPD phases. The relationship influence several aspects of services related to the product development process, scope of services, and existing infrastructure. The managerial implication of this work is the need to formally include requirements of services in the new product development since the earlier stages of this process.
\end{abstract}

Keywords: New product development. Product-related services. Manufacturing companies. After-sales services. Servitization. 


\section{Introdução}

Os serviços representam uma grande parte da economia moderna. Nos países desenvolvidos, a participação média dos serviços no PIB é de 63\%, enquanto que $68 \%$ da mão-de-obra é empregada neste setor (Central Intelligence Agency [CIA], 2008). O crescimento da participação dos serviços não está relacionado somente com os setores tradicionais de serviço tais como educação, saúde e administração financeira. Cada vez mais as empresas de manufatura estão se voltando para a oferta de serviços relacionados às suas operações e aos seus produtos (Hoffman \& Bateson, 2001; Zeithaml \& Bitner, 2003; Wilkinson \& Neely, 2009), alterando suas estratégias de modo a incluir serviços nas ofertas de produtos ao mercado (Benedettini, Swink, \& Neely 2017). A inclusão destes serviços tem sido discutida como uma proposta promissora para o crescimento das empresas, satisfação de clientes, e potencial promissor de aumento de receita e lucratividade (Eggert, Hogreve, Ulaga, \& Muenkhoff 2014, Kowalkowski, Gebauer, \& Oliva 2017).

Nesse sentido, grande parte das empresas de manufatura oferece serviços para agregar valor aos produtos, podendo se limitar a adicionar serviços diretamente associados ao produto ou mudar radicalmente o modelo de negócio da organização. Atualmente, um terço de grandes empresas tipicamente de manufatura oferece serviços (Martinez, Neely, Leinster-Evans, \& Bisessar 2017). Nessa linha, ganha força o modelo de negócios denominado de performance base contracting, que surgiu na indústria de defesa e aeroespacial e se encontra atualmente difundido na indústria de bens de consumo, como de máquinas e equipamentos (Hypko, Tilebein \& Gleich, 2010a; Hypko, Tilebein \& Gleich, 2010b). Este modelo de negócio avança na busca pela diferenciação, oferecendo não só o atendimento pós venda, como também efetu- ando o planejamento e execução da manutenção, podendo chegar a se responsabilizar pela operação (Guajardo, Cohen, Kin, \& Netessine, 2011; Alvarez, Martins \& Silva, 2015). O aumento da importância de serviços para o setor de manufatura tem sido discutido sob diversas perspectivas, dentre as quais podem ser citados argumentos econômicos, competitivos e de requisitos de mercado (Oliva \& Kallenberg, 2003, Neely, 2007; Gao, Yao, Zhu, Sun \& Lin, 2011; Smith, Maull $\& \mathrm{Ng}, 2014)$.

Nestes casos, observa-se que a gestão e os resultados dos serviços associados são influenciados pelas decisões resultantes do processo de desenvolvimento de produtos (Goffin, 2000; Markeset \& Kumar, 2003; Kumar \& Kumar, 2004; Aurich, \& Wagenknecht 2006; Wyatt, Wynn, \& Clarkson, 2009). Considerando os argumentos anteriormente descritos, que ilustram o aumento da importância assumida pelos serviços em empresas típicas de manufatura, esse trabalho objetiva analisar como os serviços são influenciados pelas atividades e resultados oriundos do processo de desenvolvimento de produto (PDP).

\section{Referencial teórico - serviços e produtos}

O foco da análise no presente trabalho é em serviços prestados para dar suporte aos produtos em uma empresa fabricante de aeronaves, abrangendo serviços requeridos pelos clientes para aquisição, instalação, uso, manutenção e descarte do produto. Diversos termos são encontrados na literatura para se referir a esses tipos de serviços (Boehm \& Thomas, 2013): serviços industriais, serviços associados a produtos, sistemas produto-serviço industriais, serviços de pós-ven$\mathrm{da}$, serviços da base instalada de produtos (Oliva \& Kallenberg, 2003), suporte ao cliente (Goffin 
\& New, 2001), serviços ao cliente (Zeithaml \& Bitner, 2003), soluções integradas (Davies, Tang, Brady, Hobday, Rush \& Gann, 2001; Windahl, 2007; Brax \& Jonsson, 2009) e serviços baseados em equipamentos (Ng, Parry, Smith, Maull, \& Briscoe, 2012). Na área de projeto e engenharia, o termo sistema produto-serviço (product-service System - PSS em inglês), tem sido um dos modelos de negócios adotados (Boehm \& Thomas, 2013), podendo ser $\mathrm{B} 2 \mathrm{~B}$ (business to business), que apresentam características industriais, e os B2C (business to consumers), diretamente ligados aos consumidores.

Conforme citado na introdução, o trabalho parte da premissa de que o processo de serviços relacionados ao produto é influenciado pelas decisões tomadas durante o processo de desenvolvimento de produtos, como destaca a literatura (e.g. Goffin, 2000; Markeset \& Kumar, 2003; Kumar \& Kumar, 2004; Aurich et al., 2006; Wyatt et al., 2009). O propósito do presente trabalho é, portanto, investigar aspectos dessa relação. Um trabalho seminal nesse sentido pode ser visto em Almeida, Cauchick Miguel e Silva (2011).

\subsection{Os serviços nas empresas de manufatura e o processo de desenvolvimento de produtos}

A oferta de serviços é um dos fatores críticos de competição e agregação de valor a um produto (Quinn, Doorley, \& Paquette, 1990; Davies, Brady, \& Hobday, 2006; Gao et al., 2011) e sua importância está associada a diversos propósitos, tais como: auxiliar na integração do cliente com o produto (Kumar \& Kumar, 2004, Pawar et al., 2009), reduzir a incerteza associada à compra do produto e aumentar a disponibilidade do produto (Araujo \& Spring, 2006); facilitar a venda de produtos; criar oportunidades de crescimento em mercados maduros e equilibrar os efeitos dos ciclos econômicos (Brax, 2005); além de diferenciar os produtos dos concorrentes e ampliar a receita (Anderson, Fornell, \& Rust 1997, Davies et al., 2006). Além dessas razões, a empresa pode se beneficiar do know-how e do acesso às informações sobre os consumidores e, com isso, ainda obter uma maior detenção do conhecimento e criar um mecanismo para obtenção das informações de uso e desempenho dos produtos. Em resumo, a oferta de serviços aproxima as empresas de manufatura de seus clientes, efetuando uma integração vertical da cadeia de produção em direção ao consumidor. Este processo é conhecido como "servitization" ou "servitização", cunhado há mais de uma década por Vandermerwe e Rada (1988). $\mathrm{O}$ processo de servitização representa um novo modelo de negócios e induz uma mudança organizacional alterando a venda de bens físicos para a uma oferta de um sistema integrado de produtos e serviços (Neely, 2007). A “servitização” não é um movimento novo, mas que está presente na consolidação das empresas no final do século XIX (Shmenner, 2009).

O desenvolvimento de um novo produto, por sua vez, pode ser representado por um processo em que o produto é concebido, projetado e lançado no mercado, o que inclui uma retro-alimentação de dados e informações tanto da produção quanto do uso do produto (Ulrich \& Eppinger, 2000). É o processo no qual ocorre a transformação de uma oportunidade de mercado em um produto disponível para venda (Krishnan \& Ulrich, 2001), por meio de um conjunto de atividades (Rozenfeld et al., 2006) executadas de maneira lógica, de forma sequencial ou simultânea (Denker, Steward, \& Browning, 2001). Os modelos iniciais de desenvolvimento de produtos definem este processo linearmente, com estágios discretos e sequenciais, ao passo que outros estudos (e.g. McCarthy, Tsinopoulos, Allen, \& Rose-Anderssen, 2006) consideram que a evolução do processo de desenvolvimento se dá através de estágios, mas com sobreposições e loops. As fases 
são determinadas pela entrega de um conjunto de resultados (Rozenfeld et al., 2006), sendo que estes irão compor os elementos do projeto do novo produto. Dentre os modelos clássicos de desenvolvimento de produtos organizado em fases, podem ser citados os de Clark e Fujimoto (1991); Cooper (2001); Ulrich e Eppinger (2000); e Rozenfeld et al. (2006).

O PDP influencia várias atividades relacionadas ao ciclo de vida do produto e dos serviços oferecidos, tais como: as necessidades de manutenção e confiabilidade do produto (Lele, 1986; Kumar \& Kumar, 2004), a quantidade de treinamento necessário e a facilidade de atualização do produ- sas. Algumas dessas perspectivas são apresentadas na sequência e foram categorizadas neste trabalho de acordo com a característica predominante, a saber: aplicação, necessidade do cliente, finalidade, natureza da interação, conteúdo, natureza das ofertas e momento da prestação do serviço. Tais categorias não são excludentes e podem ser utilizadas de forma combinada para melhor caracterizar a oferta da empresa. A combinação dessas categorias define as especificidades de cada oferta de serviços e pode servir de referência na análise das implicações dessa oferta na organização. Uma síntese da categorização utilizada é mostrada no Quadro 1. to (Goffin \& New, 2001), o suporte necessário ao item (Garvin, 1988), os meios pelos quais o produto pode ser entregue (Sleeter, 1991) e também a sua lucratividade (Knecht, Leszoinski, \& Webe 1993; Wise \& Baumgartner, 1999). Se esses elementos não forem considerados adequadamente, o desempenho dos serviços pode vir a não atender aos requisitos funcionais do produto. No entanto, apesar de os serviços serem influenciados pelo processo de desenvolvimento de produto, esta relação é pouco explorada (Markeset \& Kumar, 2003) e a literatura carece, portanto, de estudos que tratem desse assunto de maneira mais sistematizada.

\subsection{Categorização dos serviços e sua relação com o produto}

Existem diferentes perspectivas com relação à combinação de produtos e serviços pelas empre-

\begin{tabular}{|c|c|c|}
\hline Critério & Classificação na literatura & Referências \\
\hline Aplicação & $\begin{array}{l}\text { Ao consumidor } \\
\text { Industrial }\end{array}$ & $\begin{array}{l}\text { Homburg, C. e } \\
\text { Garbe; Kumar e } \\
\text { Kumar (2004) }\end{array}$ \\
\hline $\begin{array}{l}\text { Necessidades } \\
\text { do cliente }\end{array}$ & $\begin{array}{c}\text { Aquisição; Instalação; Operação; } \\
\text { Manutenção e reparos } \\
\text { Atualizações; Descarte }\end{array}$ & $\begin{array}{c}\text { Goffin e New } \\
\text { (2001); Oliva e } \\
\text { Kallenberg (2003); } \\
\text { Markeset, e Kumar } \\
\text { (2003); Gebauer et } \\
\text { al. (2008); } \\
\text { Pereira et al. (2015) }\end{array}$ \\
\hline $\begin{array}{c}\text { Finalidade das } \\
\text { ofertas }\end{array}$ & $\begin{array}{l}\text { Serviços ao cliente; Produto- } \\
\text { serviços; Serviços como um } \\
\text { produto }\end{array}$ & $\begin{array}{l}\text { Mathieu } \\
\text { (2001); Wise e } \\
\text { Baumgartner } \\
\text { (2003) }\end{array}$ \\
\hline Conteúdo & $\begin{array}{l}\text { Know-how (consultoria, treinamento } \\
\text { e suporte ao produto); Informação } \\
\text { (disponibilidade e documentação); } \\
\text { Materais (peças, equipamentos e } \\
\text { software); Manutenção (reparos, } \\
\text { atualizações e restaurações); } \\
\text { Serviços financeiros (leasing, } \\
\text { financiamento, aluguel); } \\
\text { Operação, gestão e logística } \\
\text { (instalação, planejamento, } \\
\text { integração, monitoramento, } \\
\text { distribuição, transporte) }\end{array}$ & $\begin{array}{l}\text { Vandermerwe e } \\
\text { Rada (1988); Goffin } \\
\text { (1988); Davis et } \\
\text { al. (2006); Neely } \\
\text { (2007); Visintin e } \\
\text { Rapaccini (2009) }\end{array}$ \\
\hline $\begin{array}{l}\text { Natureza da } \\
\text { integração com } \\
\text { o cliente }\end{array}$ & $\begin{array}{c}\text { Serviços baseados em transações; } \\
\text { Serviços baseados em } \\
\text { relacionamento }\end{array}$ & $\begin{array}{c}\text { Oliva e Kallenberg } \\
\text { (2003) }\end{array}$ \\
\hline
\end{tabular}

Quadro 1: Classificação das ofertas

Fonte: construída com base na literatura citada.

A partir da descrição da relevância dos serviços no contexto de empresas de manufatura e da apresentação dos diferentes tipos de serviço e 
sua classificação, a seção seguinte apresenta os métodos de pesquisa adotados para o desenvolvimento do trabalho de campo, visando alcançar seus resultados.

\section{Métodos e técnicas de pesquisa}

Yin (1994) apresenta três condições para escolha da abordagem metodológica de pesquisa: o tipo de questão de pesquisa, a extensão do controle que o pesquisador tem sobre os eventos, e o foco do trabalho de pesquisa em eventos contemporâneos ou históricos. Com base nesses critérios, com relação ao presente trabalho, pode-se dizer que a questão de pesquisa é do tipo "como", visando identificar como (e quais) os serviços fazem parte do desenvolvimento de produto, concentrando a investigação em fatos contemporâneos, sem controle sobre os eventos. Além disso, a natureza das variáveis é qualitativa também justificando, assim, a adoção da abordagem metodológica de estudo de caso como a que melhor se enquadra, em função dos propósitos do trabalho.

Para este trabalho de pesquisa foi adotado um estudo de caso único. A aplicação de casos únicos é apropriada para estudos exploratórios (Yin, 1994), uma vez que o propósito da presente pesquisa investiga um tema relativamente emergente.

Como referência para a condução do estudo de caso, foi utilizada a seguinte estrutura (Cauchick Miguel, 2007): seleção da unidade de análise e contatos, escolha dos meios para coleta e análise dos dados, desenvolvimento do protocolo para coleta e definição dos meios para análise dos dados, coleta e análise dos dados, e geração do relatório de pesquisa.

O objeto de análise escolhido deve atender os critérios de representatividade nas dimensões de interesse teórico. Embora a utilização de caso único possa ser uma limitação em termos de generalização dos resultados, o objeto de análise apresenta uma complexidade e variedade que satisfazem os propósitos da pesquisa, no que se refere ao tema de desenvolvimento de produto incorporado com serviços. Nesse sentido, foi selecionada uma empresa nacional de grande porte do setor aeronáutico, por apresentar um portfolio variado de serviços e também possuir um processo de desenvolvimento estruturado, além de o produto possuir um longo ciclo de vida e um grande número de atividades associadas à sua operação e suporte. Nesta organização, foi escolhida a unidade de negócios de aviação comercial para realização do estudo, em função de ser a unidade mais significativa da organização, representando mais de $60 \%$ da sua receita. A empresa conta com pouco mais de 19.000 funcionários no Brasil e exterior, com uma receita líquida de R\$20,3 bilhões em 2015. Já foram produzidas mais de 5.000 aeronaves que operam em quase 80 países, com um investimento em P\&D de cerca de R 350 milhões (2015). A possibilidade de amplo acesso aos dados também foi um critério contemplado para a realização do trabalho. Embora não seja decisivo, a importância e projeção da empresa no cenário econômico nacional e internacional também foram consideradas, sendo uma das maiores empresas nacionais exportadoras no país, com posicionamento significativo no exterior.

Para a coleta de dados, as seguintes fontes de evidência foram utilizadas: entrevistas semiestruturadas, entrevistas espontâneas e análise documental. A escolha da entrevista se deve ao fato da obtenção de fatos e dados que não poderiam ser coletados de outra maneira, além de possibilitar a verificação e validação desses dados em conjunto com outras fontes. A análise documental foi escolhida por ser uma fonte estável de dados e por permitir uma revisão contínua (Yin, 1994). O conteúdo do conjunto de dados foi analisado, 
remetendo a literatura quando pertinente, e pela possibilidade de comparação entre as diferentes fontes. A Figura 1 ilustra o processo de coleta e análise de dados.

Como pode ser visto na Figura 1, a coleta de dados pode ser dividida em duas etapas: (i) levantamento de dados sobre os serviços e (ii) sobre o processo de desenvolvimento de produtos. O processo de coleta iniciou com o levantamento do portfolio de serviços da empresa, realizado principalmente por meio da análise documental (documentos internos descritivos dos produtos, apresentações institucionais, catálogos de produtos, documentos descritivos de processos, entre outros documentos internos e públicos). Uma vez realizada essa etapa, foram selecionados alguns serviços para uma análise mais detalhada, principalmente, por meio de entrevistas semi-estruturadas com os responsáveis pela oferta do serviço. O objetivo dessas entrevistas foi confrontá-las com os documentos analisados, além de buscar um maior de- talhamento sobre as características dos serviços identificados. Foram também identificadas as relações preliminares entre os serviços e o PDP da empresa. Os pesquisadores utilizaram um roteiro simplificado para obter dados e informações detalhadas sobre componentes, características, e processo de produção dos serviços, além de buscar a perspectiva do entrevistado sobre a relação entre o serviço e as etapas do PDP (este roteiro é ilustrado no Apêndice 1).

O levantamento sobre o processo de desenvolvimento de produto consistiu da descrição deste processo e do detalhamento das etapas e resultados das fases do PDP. A análise foi realizada por meio de verificação de documentos descritivos dos processos e entrevistas semi-estruturadas com representantes (gerente e engenheiros) das áreas de desenvolvimento de produtos (o roteiro de entrevista pode ser visto no Apêndice 2). A partir da análise documental do PDP da empresa, foi realizada uma analogia com o modelo de referência

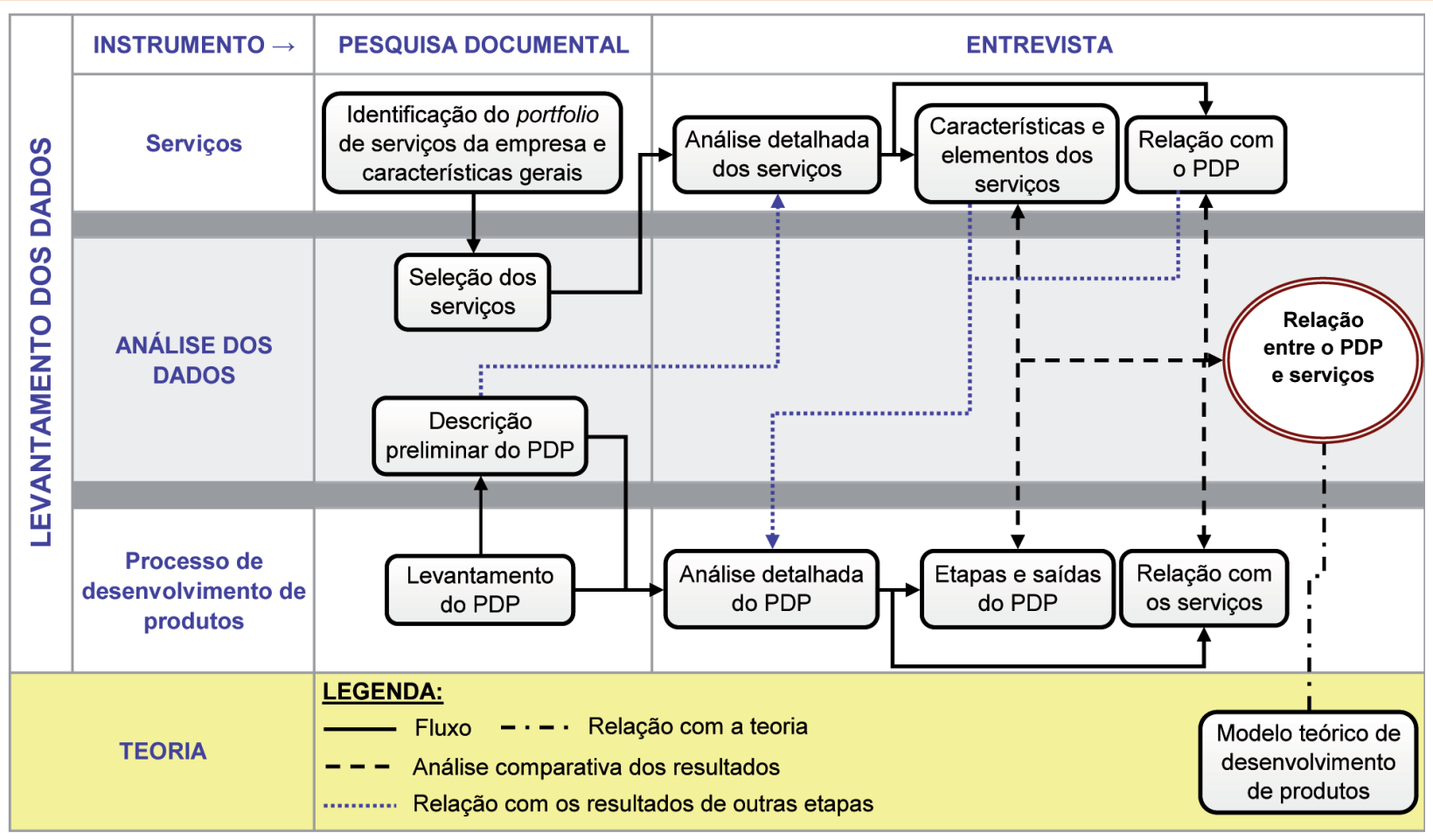

Figura 1: Coleta e análise de dados 
de Rozenfeld et al. (2006), visando identificar as similaridades entre os mesmos. Este modelo de referência foi escolhido pois é voltado para o desenvolvimento de bens de capital e é bem detalhado na literatura vigente.

A análise dos dados foi sub-dividida nas seguintes etapas: (i) análise do processo de desenvolvimento de produtos (descrição do PDP e seus resultados principais); (ii) análise dos serviços (seleção e classificação dos serviços, conforme Quadro 1); (iii) análise da relação entre o processo de desenvolvimento de produtos e os serviços; (iv) relação com a literatura vigente.

Para realizar a análise das relações entre o PDP e os serviços aplicou-se a matriz de relacionamento ilustrada na Figura 2. Nas linhas da matriz foram listados as fases e os resultados do PDP da organização e nas colunas os serviços selecionados para realizar a análise detalhada. Para cada um dos resultados do PDP, foi avaliado pelos pesquisadores qual seria a sua relação com os serviços selecionados, sendo atribuídos conceitos como "forte", "médio" ou "fraco". Com base nos resultados, foi feita uma descrição da relação de intensidade para cada serviço. No exemplo representado na Figura 2, o resultado "Desenvolvimento e liberação das publicações técnicas”, que ocorre na fase "Projeto Detalhado e Certificação", tem forte relação com o serviço de "Suporte Técnico", pois oferece material de apoio à prestação do serviço. O padrão das publicações e o meio como são disponibilizadas pode exigir a adequação dos procedimentos e dos processos do serviço de suporte técnico. Depois de completada, a matriz gerada foi posteriormente submetida para avaliação dos entrevistados.

As relações encontradas foram classificadas em quatro categorias: (i) processo, (iii) infraestrutura, (iii) pessoas e (iv) escopo. As relações que levam à adequação dos processos, procedimentos, normas, indicadores, etc., foram consi- deradas como sendo de processos. A categoria de infra-estrutura foi considerada como aquela que se relaciona com a necessidade de investimento em equipamentos, instalações, etc., enquanto que a de pessoas é aquela resultante da capacitação dos colaboradores e necessidade de treinamento. As relações que impactam o escopo do serviço foram consideradas como aquelas que se relacionam com a entrega do serviço (ou seja, com o seu conteúdo). Para finalizar (etapa iv), foi realizada uma comparação entre o PDP da empresa investigada e o modelo de referência citado anteriormente (Rozenfeld et al., 2006). Dessa forma, foi feita uma projeção dos resultados encontrados no estudo para o modelo de referência, por meio de similaridade entre as atividades do PDP da empresa e as atividades propostas em Rozenfeld et al. (2006).

\section{Resultados}

Com base na estrutura de análise dos dados apresentada na seção anterior, os resultados relativos ao PDP e os serviços da organização investigada são apresentados a seguir.

\subsection{O processo de desenvolvimento de produto da empresa investigada}

O processo de desenvolvimento de produto da empresa se baseia na abordagem de desenvolvimento integrado de produto, que integra processos, planejamento integrado, organização e o uso de ferramentas e métodos. Por meio dessa prática, busca-se integralizar o processo de desenvolvimento de produto aos demais processos de negócio da empresa, utilizando-se de times multidisciplinares e co-localizados que permitem que todos os objetos do projeto sejam integralmente considerados de forma simultânea. Cabe destacar 


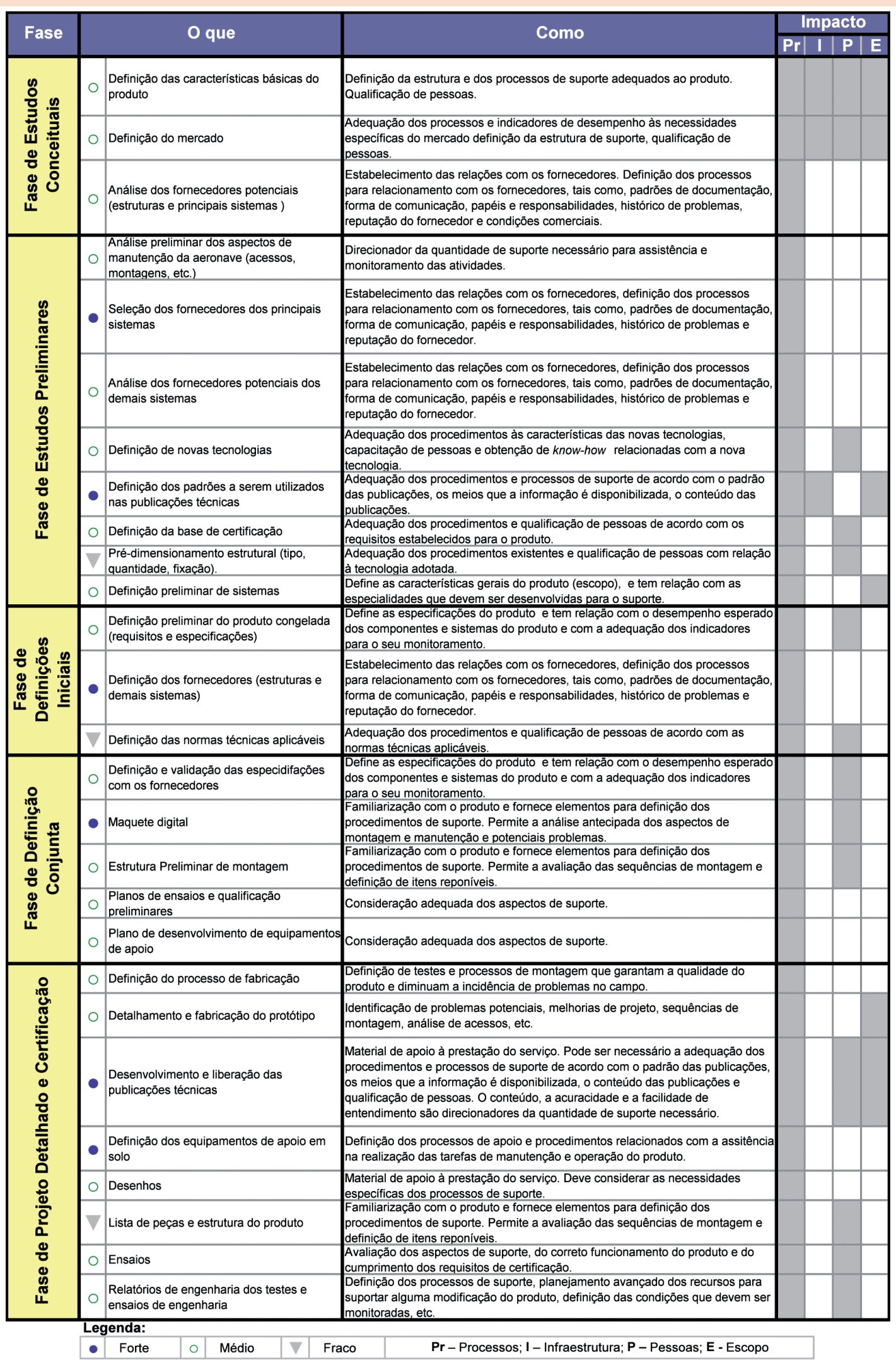

Figura 2: Matriz de avaliação do relacionamento entre o PPD e os serviços - exemplo para serviços de suporte técnico (matrizes para seis serviços diferentes). 
que, por razões restritivas em função de sigilo, alguns dados sobre o PDP foram omitidos.

Uma característica importante do processo de desenvolvimento de produtos é a participação conjunta dos fornecedores no processo de desenvolvimento dos segmentos e sistemas da aeronave. O processo de desenvolvimento da empresa é divido nas seguintes fases: (i) Estudos Conceituais, (ii) Estudos Preliminares, (iii) Definições Iniciais, (iv) Definição Conjunta, (v) Projeto Detalhado e Certificação, (vi) Seriação e (vii) Phase-out. Cada fase é composta por um conjunto de atividades e resultados que é descrito a seguir. Para realização da análise da relação entre o desenvolvimento de produto e os serviços, as fases "(vi) Seriação" e "(vii) Phase-out" foram desconsideradas, ou seja, não são descritas, uma vez que o desenvolvimento do produto em si se encerra na fase "(v) Projeto Detalhado e Certificação" e o produto já passa a ter suporte externo a partir da fase "(vi) Seriação".

$\mathrm{Na}$ fase “(i) Estudos Conceituais” novas oportunidades de negócios são identificadas pela organização e desdobradas em requisitos. São então realizadas atividades para proposição de novos produtos e, como resultado, um conceito é selecionado de maneira que atenda uma oportunidade específica. Tal conceito deve possuir um elevado potencial de aceitação, viabilidade técnica e econômica e potencial competitivo no mercado.

A fase “(ii) Estudos Preliminares” tem como objetivo definir uma configuração preliminar e assegurar a viabilidade técnica e econômica do programa (em geral, para esta empresa um programa define uma série de aeronaves a ser desenvolvida variando, principalmente, em termos de capacidade de passageiros). Esta fase envolve a estruturação do negócio e o congelamento de uma configuração base para o(s) produto(s) para detalhamento e determinação de fornecedores potenciais para os sistemas principais. Os resultados dessa etapa compreendem a descrição técnica dos itens da aeronave, especificação do desempenho dos sistemas em geral, materiais, bem como os desenhos preliminares da maquete digital (DMU - Digital Mock-up) e a definição e aprovação da base de certificação do produto.

$\mathrm{Na}$ fase “(iii) Definições Iniciais”, o planejamento do programa é preparado e o plano de negócio aprovado para início do desenvolvimento do(s) produto(s). Os principais fornecedores são selecionados nesta fase e os acordos comerciais com os mesmos são definidos e formalizados. Os principais resultados dessa fase são o plano mestre, a arquitetura do(s) produto(s), e a compilação dos seus requisitos. Os requisitos devem abranger os de engenharia, do cliente, de certificação, de suporte pós-venda, etc., sendo documentados através de um sistema de gestão de requisitos.

Na fase "(iv) Definições Conjuntas”, os fornecedores estão engajados no processo de desenvolvimento e trabalham junto da empresa no refinamento e validação da configuração inicialmente definida, complementando os dados e documentos da fase anterior. Essas definições são feitas através dos layouts de projeto, montagem e interfaces. A maquete digital e a estrutura preliminar de montagem (PSA - Preliminary Structure Analysis) são revisadas, definem-se e documentam-se os sistemas, a estrutura, ferramental, as tecnologias de produção e os processos e itens de reposição. A estrutura preliminar de montagem corresponde a um diagrama de árvore do produto que define os conjuntos e peças, instalações e a seqüência de montagem de todo o produto. São também definidas as interfaces entre as equipes de trabalho através dos Documentos de Controle de Interface (ICD - Interface Control Document). Esse documento define e controla as fronteiras dos pacotes de trabalho entre as empresas. Além das atividades mencionadas, são elaborados diversos planos para detalhamento e execução nas etapas seguintes. Dentre estes planos podem ser citados os planos de ensaios preliminares, de 
certificação, de qualificação dos processos de fabricação, plano de publicações técnicas, entre outros. Em suma, pode-se dizer que os principais resultados dessa fase são os modelos geométricos (3D), a estrutura do produto, as definições dos componentes e parâmetros do produto e os planos anteriormente citados. Além disso, ao final da fase ocorre a autorização para detalhamento do produto, descrito na sequência.

De maneira geral, pode-se dizer que a fase “(v) Projeto Detalhado e Certificação" consiste da execução e detalhamento do que foi definido nas fases anteriores, permitindo que o produto seja identificado, fabricado, inspecionado e aprovado. As atividades dessa fase podem ser divididas em três grupos principais: detalhamento, ensaios e certificação do produto. O detalhamento do produto envolve a elaboração dos desenhos, diagramas, listas de peças, modelos geométricos, roteiros de fabricação e teste, ferramentais, publicações técnicas, equipamento de apoio em solo (GSE ground support equipment), estrutura do produto, etc. Nessa fase, a aeronave protótipo e os dispositivos de ensaio são fabricados para realização dos ensaios e certificação do produto. Os ensaios têm como objetivo de garantir que os requisitos especificados serão atendidos em todas as condições operacionais identificadas. Com base nos resultados dos ensaios, são emitidos os relatórios de engenharia que comprovam o adequado funcionamento do produto. A aprovação dos relatórios e manuais pelos órgãos homologadores resulta na certificação da aeronave, com a emissão do Certificado de Homologação de Tipo (CHT). A seguir é descrita a estrutura de serviços da organização estudada.

\subsection{Os serviços e a estrutura de suporte da empresa estudada}

A empresa investigada oferece uma ampla estrutura de suporte para provisão de serviços, peças de reposição e assistência técnica. A estrutura de "Serviços ao Cliente" pode ser dividida nas seguintes categorias: "Suporte de Campo", "Suporte Técnico", "Publicações Técnicas”, "Engenharia de Manutenção", "Suporte a Operações", "Treinamento", Serviços de Manutenção", "Suporte Material” e "e-Business". A seguir é feita uma análise geral do portfolio de serviço para as categorias citadas.

Por meio da análise do portfolio de serviços foram identificados mais de 100 serviços, que incluem serviços de suporte técnico, treinamento, suporte às operações, suporte à manutenção, suporte material e logística, programas especiais de suporte, soluções de comércio eletrônico e centros de serviço, dentre outros. Do portfolio de serviços foram escolhidos seis serviços para análise detalhada. Tais serviços foram selecionados de modo a se obter uma variedade de serviços com relação as características, de modo que representasse o portolio de serviços, uma vez que não seria viável, em termos de recursos, avaliar portfólio completo de serviços. Além desse critério, foram também considerados serviços em que se pudesse se obter uma variedade em todas as categorias mostradas no Quadro 1. Os serviços selecionados foram: (A) suporte técnico; (B) publicações técnicas; (C) recomendação de aprovisionamento de peças de reposição; (D) estoque compartilhado de peças de reposição; (E) sistema de monitoramento da saúde da aeronave; $(\mathrm{F})$ manutenção de rotina. Para realização da análise detalhada foram entrevistados representantes dos departamentos de cada um dos serviços listados.

O "Suporte Técnico" consiste basicamente do apoio às necessidades técnicas do cliente no diaa-dia. De maneira geral, o escopo dos serviços de "Suporte Técnico" inclui a proposição de soluções para problemas informados pelo cliente e a identificação e monitoramento de problemas crônicos e oportunidades de melhoria de projeto do produto.

As "Publicações Técnicas" têm o objetivo de permitir que o cliente opere, mantenha e repare a 
aeronave e seus componentes de maneira segura e eficaz. As publicações podem ser divididas em publicações operacionais e publicações de manutenção, sendo que a análise detalhada foi realizada sobre as publicações de manutenção. As publicações de manutenção fornecem informações e instruções para a realização de manutenções preventivas e corretivas, manutenções programadas, planejamento de manutenção, informações sobre peças de reposição e materiais de consumo, reparo de componentes, inspeções, realização de pesquisa de panes, informações sobre equipamentos e ferramentas de apoio, recuperação de aeronave em caso de acidentes, entre outras.

O "Serviço de Recomendação de Aprovisionamento de Peças de Reposição” tem o objetivo de fornecer aos clientes uma lista detalhada das peças de reposição e equipamentos de apoio para dar suporte à operação e manutenção na fase inicial de operação do cliente. A recomendação é gerada com base na configuração e no perfil de utilização da aeronave, no número de bases de manutenção do cliente e nos lead time/turn around time das peças.

O "Estoque Compartilhado de Peças de Reposição” é um programa especial de suporte que oferece acesso ilimitado ao estoque dos componentes contratados, a gestão de reparo dos componentes inoperantes e um estoque opcional reduzido dos itens mais críticos do contrato localizado na base do cliente. O serviço oferece a cobertura dos componentes reparáveis mais críticos e demandados dos principais sistemas da aeronave.

O "Serviço de Monitoramento da Saúde da Aeronave" consiste de uma plataforma computacional para monitoramento contínuo das aeronaves da frota do cliente e fornece informações de manutenção de forma simplificada e em tempo real. As principais entregas do serviço são a exibição em tempo real de falhas, o monitoramento do local das aeronaves, a exibição de resultados de filtros pré-programados e condições de despachabilidade, o controle de atividades de pesquisa de pane e manutenção, o armazenamento do histórico de falhas do produto e a facilitação de aceso às publicações técnicas relevantes.

Os "Serviços de Manutenção de Rotina" incluem atividades de manutenção programada e não-programada, reparos estruturais e de componentes, incorporação de modificações de produto, pintura de aeronaves, inspeções, reconfiguração de aeronaves e reforma de interiores, pesagem de aeronaves, dentre outras. A análise detalhada foi realizada sobre as atividades de manutenção programada. Essas atividades correspondem àquelas necessárias para manter a aeronave aeronavegável, de acordo com o plano de manutenção do cliente aprovado junto à agência reguladora local. De maneira geral, o intervalo para realização das tarefas pode estar vinculado ao tempo (meses, dias), à utilização do produto (horas de utilização, ciclos, ou à combinação de ambos).

O Quadro 2 apresenta uma síntese dos principais elementos dos serviços selecionados. Uma classificação desses serviços (de acordo com os critérios do Quadro 1), é mostrada no Quadro 3

A partir da descrição dos serviços e sua respectiva classificação, a seção seguinte apresenta os resultados da aplicação da matriz de relacionamento entre os serviços e o processo de desenvolvimento de produtos da empresa (Figura 2).

\subsection{A relação entre os serviços e o processo de desenvolvimento de produtos}

Esta seção apresenta os resultados da aplicação da matriz de relacionamento. A Figura 3 mostra uma visão geral das relações encontradas entre os serviços e o PDP pela aplicação da matriz de relacionamento e que são descritas na sequência. A figura ilustra as fases de desenvolvimento de produto (primeira coluna) associado aos serviços sele- 


\begin{tabular}{|c|c|}
\hline Serviços & Principais elementos \\
\hline Suporte Técnico & $\begin{array}{c}\text { Mão de obra especializada de engenharia } \\
\text { Histórico de problemas técnicos do produto } \\
\text { Manuais e desenhos de engenharia } \\
\text { Requisitos de homologação } \\
\text { Normas técnicas }\end{array}$ \\
\hline $\begin{array}{l}\text { Publicações } \\
\text { Técnicas }\end{array}$ & $\begin{array}{c}\text { Informações técnicas } \\
\text { Procedimentos associados à manutenção e à } \\
\text { utilização da aeronave } \\
\text { Procedimentos relacionados às atividades de } \\
\text { suporte e à utilização da aeronave } \\
\text { Integração e disponibilidade das informações }\end{array}$ \\
\hline $\begin{array}{l}\text { Recomendação de } \\
\text { Aprovisionamento } \\
\text { de Peças de } \\
\text { Reposição }\end{array}$ & $\begin{array}{c}\text { Configuração do produto } \\
\text { Dados de confiabilidade dos componentes } \\
\text { Custos e preços dos componentes } \\
\text { Turn around time e lead time } \\
\text { Relações com o perfil de utilização da aeronave }\end{array}$ \\
\hline $\begin{array}{c}\text { Estoque } \\
\text { Compartilhado } \\
\text { de Peças de } \\
\text { Reposição }\end{array}$ & $\begin{array}{c}\text { Configuração do produto } \\
\text { Dados de confiabilidade dos componentes } \\
\text { Custos e preços dos componentes } \\
\text { Turn around time e lead time } \\
\text { Relações com o perfil de utilização da aeronave } \\
\text { Custos de reparo dos componentes } \\
\text { Base de clientes }\end{array}$ \\
\hline $\begin{array}{l}\text { Sistema de } \\
\text { Monitoramento } \\
\text { da Saúde da } \\
\text { Aeronave }\end{array}$ & $\begin{array}{c}\text { Sistema instalado na aeronave para geração e } \\
\text { transmissão das mensagens } \\
\text { Sistema de solo para recepção e roteamento dos } \\
\text { dados enviados para a aeronave } \\
\text { Sistema de solo para tratamento dos dados } \\
\text { recebidos } \\
\text { Sistema de solo para exibição dos dados } \\
\text { Equipe de suporte ao usuário }\end{array}$ \\
\hline $\begin{array}{l}\text { Manutenção de } \\
\text { rotina }\end{array}$ & $\begin{array}{c}\text { Pessoal qualificado } \\
\text { Peças e materiais disponíveis } \\
\text { Equipamento de apoio para realização das tarefas } \\
\text { Manuais técnicos } \\
\text { Instalações } \\
\text { Manual de processos da empresa }\end{array}$ \\
\hline
\end{tabular}

Quadro 2: Principais elementos dos serviços selecionados

Fonte: documentos internos da organização e entrevistas semi-estruturadas. de ("forte", "médio", "fraco" ou "inexistente").

Conforme pode ser visto na Figura 4, os serviços são influenciados por atividades que estão presentes em todas as fases de desenvolvimento de produto analisadas. Pela matriz de relacionamento, também foi identificado que os resultados do PDP influenciam os serviços selecionados. No entanto, a intensidade desse relacionamento pode diferir de um serviço para outro, dependendo da sua característica.

A Figura 4 também mostra que não foi identificada nenhuma relação "forte" na fase inicial de "Estudos Conceituais". No entanto, nota-se que a ocorrência dessa relação é crescente nas fases seguintes. Isso pode ocorrer devido ao fato de que as atividades executadas nas fases iniciais do desenvolvimento do produto são definições mais gerais, sendo mais específicas/detalhadas nas fases subsequentes. De modo análogo, nota-se que a maioria das relações encontradas é "média”, o que também pode ser visto na Figura 4. Este resultado também é mostrado na matriz de relacionamento da Figura 3, tendo identificado que os resultados do PDP influenciam diversos serviços. No entanto, esse relacionamento pode diferir de um serviço para outro, dependendo das características do serviço. Por exemplo, a definição dos equipamentos de apoio influencia na definição dos processos de suporte técnico, pois os equipamentos de apoio são parte dos procedimentos definidos nas publicações técnicas e o conteúdo de algumas publicações é parte do conteúdo das re- 


\begin{tabular}{|c|c|c|c|c|}
\hline Serviços & Necessidades do cliente & Finalidade & Conteúdo & $\begin{array}{l}\text { Natureza de interação } \\
\text { com o cliente }\end{array}$ \\
\hline (A) Suporte técnico; & $\begin{array}{c}\text { Manutenção e reparo/ } \\
\text { atualizações }\end{array}$ & $\begin{array}{c}\text { Serviços ao } \\
\text { cliente/produto- } \\
\text { serviços }\end{array}$ & Know-how & $\begin{array}{l}\text { Serviços baseados em } \\
\text { relacionamento }\end{array}$ \\
\hline (B) Publicações técnicas & $\begin{array}{c}\text { Instalação/manutenção } \\
\text { e reparo/atualizações }\end{array}$ & $\begin{array}{c}\text { Serviços ao } \\
\text { cliente/produto- } \\
\text { serviços }\end{array}$ & Informação & $\begin{array}{l}\text { Serviços baseados em } \\
\text { relacionamento }\end{array}$ \\
\hline $\begin{array}{l}\text { (C) Recomendação de } \\
\text { aprovisionamento de } \\
\text { peças de reposição }\end{array}$ & $\begin{array}{c}\text { Aquisição/instalação/ } \\
\text { manutenção e reparo/ } \\
\text { atualizações }\end{array}$ & $\begin{array}{l}\text { Serviços ao } \\
\text { cliente }\end{array}$ & Know-how & $\begin{array}{l}\text { Serviços baseados em } \\
\text { relacionamento }\end{array}$ \\
\hline $\begin{array}{c}\text { (D) Estoque } \\
\text { compartilhado de } \\
\text { peças de reposição }\end{array}$ & $\begin{array}{l}\text { Instalação/manutenção } \\
\text { e reparo/atualizações }\end{array}$ & Produto-serviços & $\begin{array}{l}\text { Materiais, } \\
\text { serviços } \\
\text { financeiros }\end{array}$ & $\begin{array}{l}\text { Serviços baseados em } \\
\text { relacionamento }\end{array}$ \\
\hline $\begin{array}{c}\text { (E) Sistema de } \\
\text { monitoramento da } \\
\text { saúde da aeronave }\end{array}$ & $\begin{array}{c}\text { Operação/manutenção } \\
\text { e reparo }\end{array}$ & Produto-serviços & $\begin{array}{c}\text { Operação, } \\
\text { gestão e } \\
\text { logística, } \\
\text { informação }\end{array}$ & $\begin{array}{l}\text { Serviços baseados em } \\
\text { relacionamento }\end{array}$ \\
\hline $\begin{array}{l}\text { (F) Manutenção de } \\
\text { rotina }\end{array}$ & $\begin{array}{l}\text { Manutenção e reparos/ } \\
\text { atualizações/ descarte }\end{array}$ & $\begin{array}{c}\text { Produto-serviços/ } \\
\text { serviços como um } \\
\text { produto }\end{array}$ & Manutenção & $\begin{array}{l}\text { Serviços baseados em } \\
\text { relacionamento/ serviços } \\
\text { baseados em transações }\end{array}$ \\
\hline
\end{tabular}

Quadro 3: Classificação dos serviços selecionados

Fonte: documentos internos da organização e entrevistas semi-estruturadas.

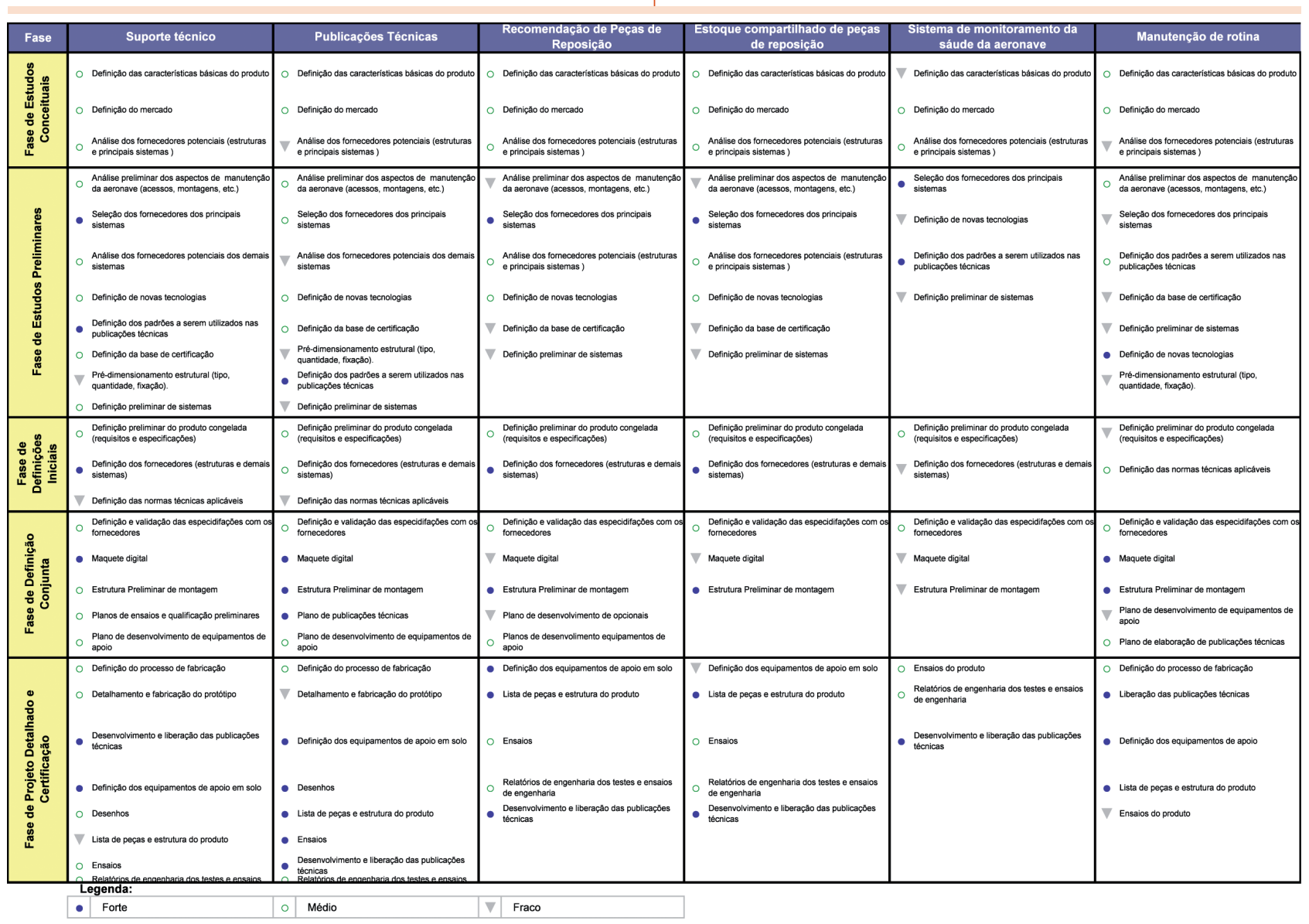

Figura 3: Resultados da matriz de relacionamento entre o PDP e os serviços. 


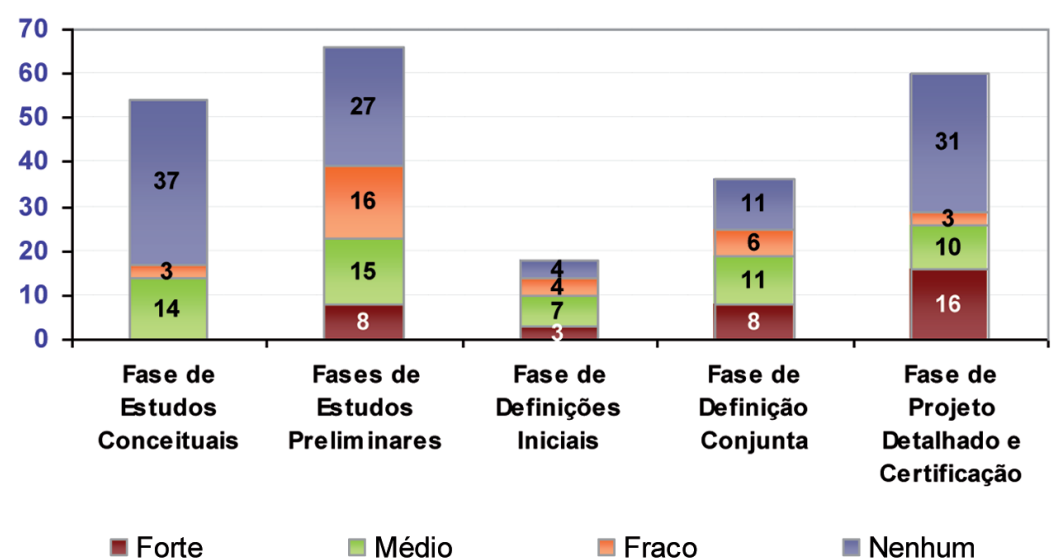

(a) Quantidade de serviços

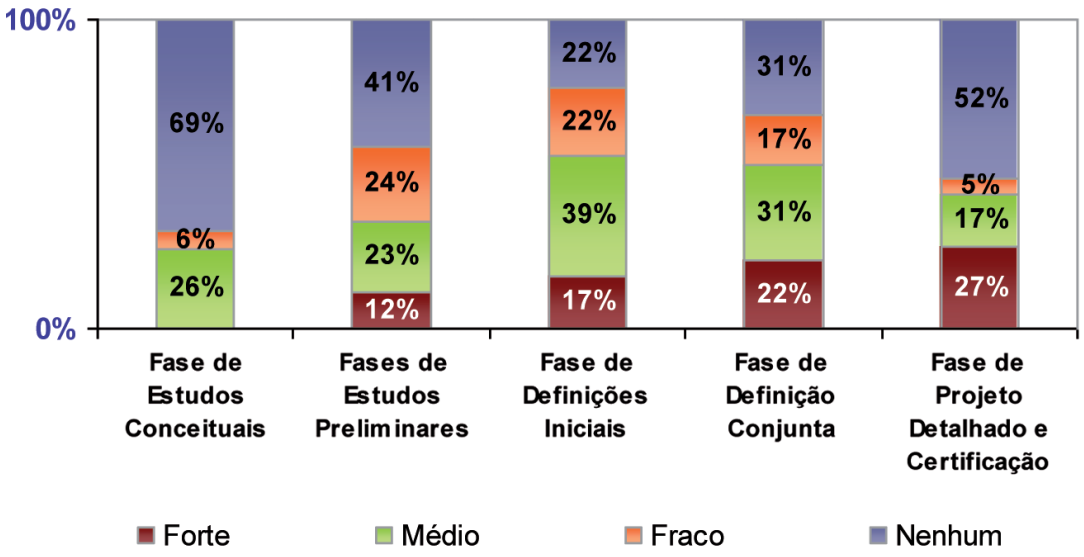

(b) Percentual de serviços

Figura 4: Relação dos serviços com o PDP por Fase do PDP

comendações de peças de reposição e equipamentos de apoio e são recursos utilizados na realização das tarefas de manutenção programada.

Da mesma forma que todas as fases do processo de desenvolvimento de produto possuem alguma relação com os serviços, a Figura 5 mostra que todos os serviços analisados são afetados pelas definições e resultados do PDP.

A Figura 5 mostra que os serviços de suporte técnico e de publicações técnicas foram os que mais apresentaram relações com o PDP (identificado pelas relações "forte" mais "médio"). Uma das serviço possíveis razões seria o fato desses serviços não serem muito específicos - não são relacionados a alguma atividade específica, como manutenção ou planejamento e, dessa forma, possuem relação com diversas atividades relacionadas ao uso e suporte do produto.

Dos resultados do PDP (considerando todas as saídas do PDP), $72 \%$ mostraram alguma relação com os serviços analisados, sendo que $10 \%$ dos resultados do PDP possuem um relacionamento com todos os serviços analisados. Dentre os resultados que possuem relacionamento com todos os serviços estão: desenvolvimento e liberação das publicações técnicas, seleção de fornecedores, maquete digital, estrutura do produto e lista de peças, definição de novas tecnologias, ensaios do produto e as definições das especificações e características do produto presentes nas diversas fases (definição das características básicas do produto, definição preliminar de sistemas, defini-
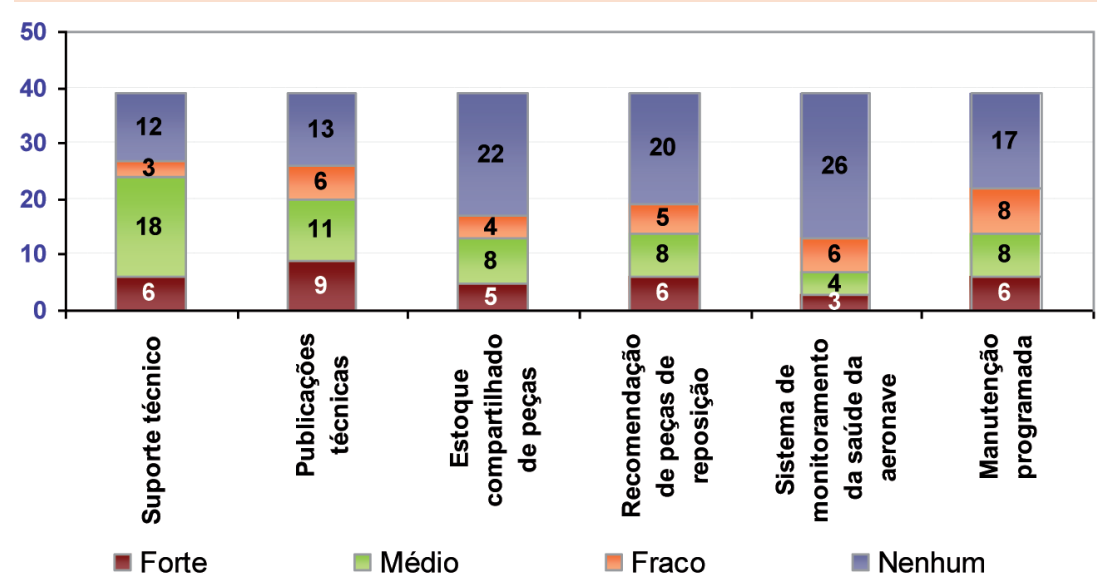

Figura 5: Relação dos serviços com o PDP de acordo com o tipo 
ção preliminar do produto congelada, definição e validação das especificações com fornecedores).

A Figura 6 mostra que, do total de relacionamentos analisados (serviços versus resultados do PDP), mais da metade possui algum relacionamento com os serviços, sendo que: quase um quarto possui um relacionamento "moderado", com o restante distribuído nas outras relações de intensidade. Considerando a ponderação dos resultados da matriz de relacionamento (soma de todos os pesos atribuídos aos relacionamentos), têm-se que as atividades e resultados do PDP que mais apresentaram relação com os serviços analisados foram: “definição e liberação das publicações técnicas"; "seleção dos fornecedores"; "estrutura do produto e lista de peças"; "definição dos equipamentos de apoio"; "maquete digital”; "definição de novas tecnologias"; "ensaios do produto"; "definição e validação das especificações com fornecedores”; "definição das características básicas do produto"; "definição preliminar do produto congelada"; "posicionamento no mercado"; "relatórios de engenharia"; "análise dos aspectos de manutenção".

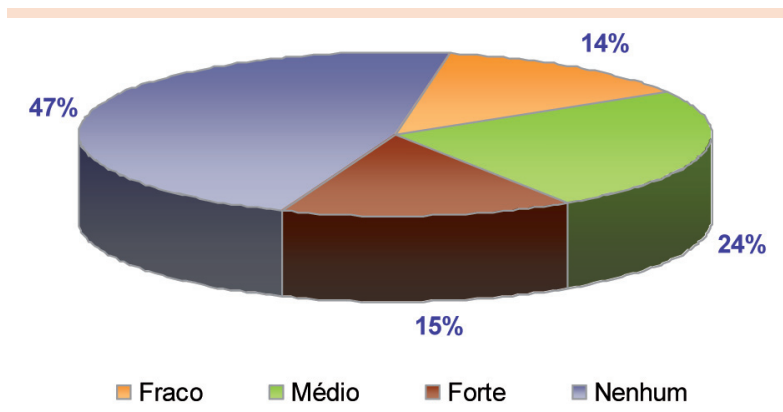

Figura 6: Distribuição do total de relações entre o PDP e serviços

\subsection{Síntese da discussão dos resultados}

Os resultados do presente trabalho evidenciam algumas das afirmações encontradas na literatura sobre a relação das decisões e resultados do PDP com os serviços. Uma delas é de que o desenvolvimento do produto influencia a quantidade de suporte necessário e os meios que ele pode ser entregue, já delineado por Garvin (1988) e Sleeter (1991) há algum tempo. Além deste, incluem-se as necessidades de manutenção e confiabilidade do produto (Lele, 1986; Kumar \& Kumar, 2004) e a facilidade de atualização do produto (Goffin \& New, 2001). Também se verifica que a análise da arquitetura do produto deve considerar os aspectos do ciclo de vida do produto nos estágios iniciais do processo de desenvolvimento (Wyatt et al., 2009). Além das atividades que são citadas na literatura, foram também identificadas implicações mais evidentes, como as relacionadas aos processos necessários para prestação e desenvolvimento dos serviços, nos custos do serviço e de sua implantação, nas necessidades de qualificação de pessoas, na definição do escopo dos serviços, nas limitações de escopo decorrentes da não-consideração dos seus requisitos, na qualidade do serviço, nos riscos associados com a oferta dos serviços, dentre outros.

Considerando o que foi exposto anteriormente, foi confirmada que existe a necessidade de integração dos requisitos dos serviços ao processo de desenvolvimento de produto, como já destacado por Goffin e New (2001). Ainda alinhado com os autores citados, pode-se também afirmar que, além da consideração nos momentos iniciais do PDP, a avaliação dos requisitos dos serviços deve ser feita de maneira abrangente. Em suma, os resultados descritos nessa seção mostram que os serviços estão relacionados com a definição do PDP, em diferentes momentos (fases) do PDP.

\section{Conclusões}

A realização do estudo descrito nesse trabalho permitiu a análise de um portfolio de serviços relacionados a produtos. A análise da relação entre o PDP e os serviços confirmou que os serviços são influenciados pelas definições realizadas 
durante o PDP e ressalta a importância da consideração dos requisitos dos serviços nesse processo. Foi identificado que essas relações ocorrem durante praticamente todas as fases de desenvolvimento do produto e estão relacionadas com as definições do PDP referentes ao produto, ao processo de fabricação, aos testes e ensaios, aos processos de apoio e aos serviços em si. As relações identificadas influenciam diversos aspectos dos serviços, tais como: os processos para prestação e desenvolvimento dos serviços, os custos e preços dos serviços, a quantidade de suporte necessário, os riscos associados com a prestação do serviço, a necessidade de qualificação de pessoas, a necessidade de investimentos em infra-estrutura, o escopo e diversidade dos serviços. Sendo assim, considera-se importante levar formalmente em conta os requisitos dos serviços desde as fases iniciais do processo de desenvolvimento e a criação de ferramentas que garantam que esses requisitos sejam atendidos ou que permitam a análise dos impactos do seu não cumprimento.

Explicitar estas relações é importante para fazer avançar a teoria no que concerne ao projeto do produto em empresas servitizadas, tanto do ponto de vista acadêmico quanto gerencial, e é esta a principal contribuição deste artigo.

Como qualquer trabalho de pesquisa este apresenta algumas limitações. A utilização de um estudo de caso único apresenta limitações com relação à validade externa (generalização para outros segmentos, produtos ou empresas). No entanto, a diversidade de serviços da unidade selecionada e a análise de múltiplos serviços fornecem elementos necessários para a análise realizada no presente trabalho. Outro aspecto que deve ser considerado é a natureza restrita das informações da empresa, em função de sigilo requerido, que implicou em uma menor possibilidade de detalhamento descritivo e analítico do caso, porém suficiente para o cumprimento dos objetivos estabelecidos.
As recomendações para futuras pesquisas são resultado tanto das limitações descritas anteriormente, quanto das lacunas identificadas durante desenvolvimento do trabalho. Para continuidade, pretende-se: analisar a integração dos serviços na concepção do desenvolvimento de produto, especialmente considerando novos serviços para empresas de manufatura, a partir dos requisitos necessários para tal incorporação. Alinhado com esta possibilidade, pode-se ainda analisar os diferentes tipos de serviço aplicáveis aos produtos a partir das necessidades dos clientes.

\section{Agradecimentos}

Os autores agradecem as recomendações e sugestões dos revisores para aprimoramento desse manuscrito. Um dos autores tem vínculo formal com a Universidade Federal de Santa Catarina e, por esta razão, a instituição também merece agradecimentos.

\section{Referências}

Alvarez, R. LP, Martins, M. R. \& Silva, M. T. (2015). Applying the maturity model concept to the servitization process of consumer durables companies in Brazil. Journal of Manufacturing Technology Management, 26(8), p. 1086-1106.

Almeida, L., Cauchick Miguel, P.A. \& Silva, M.T. (2011). Uma revisão da literatura sobre "servitização" como base para a proposição de um modelo conceitual de decisão. Exacta, 9(3), 339-354. https://doi. org/10.5585/exacta.v9i3.3115

Anderson, E. W., Fornell, C., \& Rust, R. T. (1997). Customer satisfaction, productivity, and profitability: Differences between goods and services. Marketing Science, 16(2), 129-145.

Araujo, L., \& Spring, M. (2006). Services, products, and the institutional structure of production. Industrial Marketing Management, 35(7), 797-805.

Aurich, J.C., Fuchs, C., \& Wagenknecht, C. (2006). Life cycle oriented design of technical product-service systems. Journal of Cleaner Production, 14(17), 1480-1494. 
Benedettini, O., Swink, M. \& Neely, A. (2017). Examining the influence of service additions on manufacturing firms' bankruptcy likelihood. Industrial Marketing Management, 60, 112-125.

Boehm, M., \& Thomas, O. (2013). Looking beyond the rim of one's teacup: a multidisciplinary literature review of Product-Service Systems in Information Systems, Business Management, and Engineering \& Design. Journal of Cleaner Production, 51, 245-260.

Brax, S. (2005). A manufacturer becoming service provider - challenges and a paradox. Management Service Quality, 15(2), 142-155.

Brax, S.A., \& Jonsson, K. (2009). Developing integrated solution offerings for remote diagnostics: a comparative case study of two manufacturers. International Journal of Operations and Production Management, 29(5), 539-560.

Cauchick Miguel, P. A. (2007). Estudo de caso na engenharia de produção: estruturação e recomendações para a sua conclusão. Produção, 17(1), 216-229.

CIA - Central Intelligence Agency. The 2008 world factbook. Retrieved in June 5, 2008, from https://www. cia.gov/library/publications/the-world-factbook.

Clark, K.B., \& Fujimoto, T. (1991). Product development performance: strategy, organization and management in the world auto industry. Boston: Harvard Business School Press. 405 p.

Cooper, R.G. Winning at new products-accelerating the process from idea to lauch (3a. ed.). Cambridge: Perseus Publishing, 2001.

Davies, A., Brady, T., \& Hobday, M. (2006). Charting a path toward integrated solutions. Sloan Management Review, 47(3), 39-48.

Davies, A., Tang, P., Brady, T., Hobday, M., Rush, H., \& Gann, D. (2001). Integrated solutions: The new economy between manufacturing and services. Brighton: SPRU.

Denker, S., Steward, D.V., \& Browning, T.R. Planning concurrency and managing iteration in projects. Project Management Journal, 32(3), 31-38.

Eggert, A., Hogreve, J., Ulaga, W. \& Muenkhoff, E. (2014). Revenue and profit implications of Industrial Service Strategies. Journal of Service Research, 17(1), 23-39.

Gao, J., Yao, Y., Zhu, V.C.Y., Sun, L., \& Lin, L. (2011). Service-oriented manufacturing: A new product pattern and manufacturing paradigm. Journal of Intelligent Manufacturing, 22(3), 435-446.

Garvin, D. A. (1988). Managing quality: the strategic and competitive edge. New York: The Free Press.

Goffin, K. (1998). Evaluating customer support during new product development - an exploratory study. Journal of Product Innovation Management, 15(1), 42-56.

Goffin, K. (2000). Design for supportability: essential component of new product development. Research Technology Management, 43(2), 40-47.
Goffin, K., \& New, C. (2001). Customer support and new product development - an explanatory study. International Journal of Operations \& Production Management, 21(3), 275-301.

Guajardo, J. A., Cohen, M., Kin, S., \& Netessine, S. (2001). Impact of performance-based contracting on product reliability: an empirical analysis. INSEAD working paper no. 2011/49/TOM, 2011.

Hoffman, K. D., \& Bateson, J. (2001). Marketing de serviços. São Paulo: Bookman.

Homburg, C., \& Garbe, B. (1999). Towards an improved understanding of industrial services: quality dimensions and their impact on buyer-seller relationships. Journal of Business-to-Business Marketing, 6(2), 39-71.

Hypko, P., Tilebein, M. e Gleich, R. (2010b). Benefits and uncertainties of performance-based contracting in manufacturing industries. Journal of Service Management, 21(4), 460-489.

Hypko, P., Tilebein, M., \& Gleich, R. (2010a). Clarifying the concept of performance-based contracting in manufacturing industries. Journal of Service Management, 21(5), 625-655.

Knecht, T., Leszoinski, R., \& Webe, F.A. (1993). Making profits after the sale. The McKinsey Quarterly, 4, 79-86.

Kowalkowski, C., Gebauer, H. and Oliva, R. (2017). Service growth in product firms: Past, present, and future, Industrial Marketing Management, 60, 82-88.

Krishnan, V., \& Ulrich, K. T. (2001). Product development decisions: a review of the literature. Management Science, 47(1), 1-21.

Kumar, R., \& Kumar, U. (2004). A conceptual framework for the development of a service delivery strategy for industrial systems and products. Journal of Business \& Industrial Marketing, 19(5), 310-319.

Lele, M.M. (1986) How service needs influence product strategy, Sloan Management Review, 28(1), 63-70.

Markeset, T., \& Kumar, U. (2003). Design and development of product support and maintenance concepts for industrial systems. Journal of Quality in Maintenance Engineering, 9(4), 376-392.

Martinez, V., Neely, A., Velu, C., Leinster-Evans, S. \& Bisessar, D. (2017). Exploring the journey to services. International Journal of Product Economics, 192, 66-80.

Mathieu, V. (2001). Product services: from a service supporting the product to a service supporting the client. Journal of Business \& Industrial Marketing, 16(1), 39-58.

McCarthy, I.P., Tsinopoulos, C., Allen, P., \& RoseAnderssen, C. (2006). New product development as a complex adaptive system of decisions. Journal of Product and Innovation Management, 23(5), 437-456.

Neely, A. (2007). The servitization of manufacturing: an analysis of global trends. Proceedings of the POMS College of Service Operations and EurOMA Conference, Londres, UK. 
Ng, I., Parry, G., Smith, L., Maull, R., \& Briscoe, G. (2012). Transitioning from a goods-dominant to a servicedominant logic: Visualising the value proposition of RollsRoyce. Journal of Service Management, 23(3), 416-439.

Oliva, R., \& Kallenberg, R. (2003). Managing transition from products to services. International Journal of Service Industry Management, 14(2), 160-72.

Pawar, K. S. et al. (2009). The PSO triangle: Designing product, service and organisation to create value. International Journal of Operations and Production Management, 29(5), 468-493.

Quinn, J.B., Doorley, T.L., \& Paquette, P.C. (1990). Beyond products: service-based strategy, Harvard Business Review, 68(2), 58-67.

Rozenfeld, H. et al. (2006). Gestão do Desenvolvimento de produtos - uma referência para a melhoria do processo. São Paulo: Saraiva.

Shmenner, R.W. (2009). Manufacturing, service, and their integration: Some history and theory. International Journal of Operations and Production Management, 29(5), 431-443.

Sleeter, M. (1991). How product usability impacts the service organization, AFSM International - The Professional Journal, 16(2), 59-61.

Smith, L., Maull, R., \& Ng, I.CL. (2014) Servitization and operations management: a service dominant-logic approach. International Journal of Operations \& Production Management, 34(2), 242-269.

Ulrich, K.T., \& Eppinger, S.D. (2000). Product design and development. (2a ed.). McGraw-Hill.

Vandermerwe, S., \& Rada, J. (1988). Servitization of business: adding value by adding services. European Management Journal, 6(4), 314-24.

Visintin, F., Rapaccini, M. (2009). A theoretical framework for developing product-service strategies. Proceedings of the Annual Conference of the Production and Operations Management Society, Orlando, FL, USA, 20.

Wilkinson, A.D., \& Neely, A. (2009). Changing times and changing times scales: the servitization of manufacturing. International Journal of Operations and Production Management, 29(5). (Guest Editorial).

Windahl, C. (2007). Integrated solutions in the capital goods sector. Exploring innovation, service and network perspectives. Tese de doutorado, Instituto de Tecnologia, Linköping University, Linköping.
Wise, R., \& Baumgartner, P. (1999). Go downstream: the new imperative in manufacturing. Harvard Business Review, 77(5), 133-41.

Wyatt, D.F., Wynn, D.C., \& Clarkson, J. (2009). Comparing representations for product architecture design through life-cycle evaluation methods. Proceedings of the Nordic Conference on Product Life Cycle Management, Göteborg, Suécia.

Yin, R.K. (1994). Case study research. Design and methods (2a. ed.). Thousand Oaks: Sage Publication.

Zeithaml, V.A., \& Bitner, M.J. (2003). Services marketing. integrating customer focus across the Firm (3a ed.) Boston: McGraw-Hill Higher Education.
FORMULÁRIO PARA ANÁLISE DOS SERVIÇOS

\section{Entrevistado:}

Posição:

Serviço:

Classificação do serviço (validação):

Descrição do serviço:

Componentes do serviço:

\section{Características gerais:}

Processo de entrega do serviço:

Relação com o processo de desenvolvimento de produtos: Etapa 1:

Etapa 2:

Etapa 3:

Etapa 4

Etapa $n:$

Apêndice 1: Formulário para registro e análise dos serviços e sua relação com o PDP 


\section{FORMULÁRIO PARA ANÁLISE DO PDP}

\begin{tabular}{l}
\hline Entrevistado: \\
\hline Posição: \\
\hline Etapa do processo de desenvolvimento de produtos \\
\hline
\end{tabular}

Descrição da etapa:

Principais atividades e saídas:

Relação com os serviços:

Serviço 1:

Serviço 2:

Serviço 3:

Serviço 4:

Serviço n:

Apêndice 2: Formulário para registro e análise do PDP e relação com os serviços

Recebido em 21 jun. 2017 / aprovado em 14 dez. 2017

Para referenciar este texto

Almeida, L., Silva, M. T., \& Cauchick-Miguel, P. A.

Servitização em uma empresa fabricante de aeronaves: a relação dos serviços com o desenvolvimento de produto. Exacta, São Paulo, v. 16, n. 4, p. 59-77. out./dez. 2018. Disponível em: <https://doi.org/10.5585/

ExactaEP.v16n4.7527> 
H..... . . . 